\title{
BMJ Open Survey on self-reported psychotropic drug prescribing practices of eating disorder psychiatrists for the treatment of young people with anorexia nervosa
}

\author{
Maedeh Y Beykloo, ${ }^{\oplus}$ Dasha Nicholls, ${ }^{2}$ Mima Simic, ${ }^{3}$ Ruth Brauer, ${ }^{\oplus 1}$ \\ Elizabeth Mills, ${ }^{1}$ Ian C K Wong ${ }^{1,4}$
}

To cite: Y Beykloo M, Nicholls D, Simic M, et al. Survey on selfreported psychotropic drug prescribing practices of eating disorder psychiatrists for the treatment of young people with anorexia nervosa. BMJ Open 2019;9:e031707. doi:10.1136/ bmjopen-2019-031707

\section{- Prepublication history and} additional material for this paper are available online. To view these files, please visit the journal online (http://dx.doi. org/10.1136bmjopen-2019031707).

Received 16 May 2019 Revised 01 August 2019 Accepted 14 August 2019

Check for updates

(C) Author(s) (or their employer(s)) 2019. Re-use permitted under CC BY-NC. No commercial re-use. See rights and permissions. Published by BMJ.

${ }^{1}$ Research Department of Practice and Policy, UCL School of Pharmacy, University College London, London, UK

${ }^{2}$ Faculty of Medicine, Department of Brain Sciences, Imperial College London,

London, UK

${ }^{3}$ Michael Rutter Centre, South London and Maudsley NHS

Foundation Trust, London, UK

${ }^{4}$ Department of Pharmacology and Pharmacy, University of

Hong Kong, Hong Kong, Hong Kong

Correspondence to

Dr lan C K Wong;

wongick@hku.hk

\section{ABSTRACT}

Objectives To survey current prescribing practices of psychotropic drugs by child and adolescent eating disorder (CAED) psychiatrists in the treatment of anorexia nervosa (AN).

Design Cross-sectional self-administered survey. Setting All children and young people eating disorder services (CYP EDS) in England during a national training programme.

Participants 44 CAED psychiatrists practising in CYP EDS in England.

Primary and secondary outcome measures CAED psychiatrists completed a questionnaire regarding the pattern of psychopharmacological care in AN that they provide and the medication treatment pattern at their CYP EDS. Secondary outcome measures included the process of continuing pharmacotherapy from secondary care to primary care.

Results Of the 77 CYP EDS representing every team in England, 44 teams represented by a CAED psychiatrist responded, despite 13 having no psychiatrists in post at the time of the study (response rate 69\%). Most (40\%) respondents estimated that $<10 \%$ of patients with AN were prescribed psychotropic medications. Olanzapine was reported as the most commonly prescribed medication for AN by $38 \%$ of the respondents, followed by fluoxetine (29\%) and sertraline (10\%). The most common minimum olanzapine initiation dose in this study was at $2.5 \mathrm{mg} /$ day for a duration of 2-4 weeks, reaching a maximum dose of $5 \mathrm{mg} /$ day. Most $(68 \%)$ reviewed medications every week (30\%) or every 2 weeks $(38 \%)$. Over $50 \%$ of the respondents reported continuation of olanzapine prescribing within the CYP EDS teams.

Conclusions This nationally representative survey showed that despite a lack of evidence, psychotropic medications are commonly prescribed to a minority of patients, most frequently, olanzapine. Further evidence is needed on which patients may potentially benefit from pharmacotherapy as an adjunct to psychological interventions.

\section{INTRODUCTION}

Anorexia nervosa (AN) is a psychiatric condition characterised by severe weight loss, fear of weight gain and body shape conflicts ${ }^{1}$ that

\section{Strengths and limitations of this study}

- First questionnaire study to address pharmacotherapy approach in anorexia nervosa (AN) in young people.

- This study included all child and young people eating disorder services in England.

- This study is based on real-life clinical practice, which may differ between services. However, it was unable to investigate why psychotropic medications are prescribed without any evidence to support practices.

- This study was unable to investigate barriers to and limitations and enhancing factors of pharmacotherapy in young people with AN.

poses a risk of physical, social and emotional impairments, particularly in the adolescent population. ${ }^{2}$ This results in restrictions in eating, purging or fasting, and excessive exercise. ${ }^{3}$ In instances where a potential diagnosis of $\mathrm{AN}$ is made by a healthcare professional in a primary healthcare setting, the National Institute for Health and Care Excellence (NICE) guideline ${ }^{4}$ recommends the involvement of a physician with an appropriate specialty in the treatment of AN. Patients with AN are recommended by NICE to be under the care of secondary care services, such as dedicated children and young people eating disorder services (CYP EDS). Most dedicated CYP EDS offer evidence-based psychological interventions as the primary intervention, delivered within the context of a multidisciplinary team (MDT). One of the roles of the MDT is to recognise and manage complications associated with AN, such as medical instability and comorbidities. In order to better understand the treatment provided by child and adolescent eating disorder (CAED) psychiatrists, it 
is important to explore medication prescribing practices for the treatment of individuals with AN.

During the past decade, psychotropic medications have played a notable role in the management of some psychiatric disorders in children and adolescents, ${ }^{2}$ predominantly stimulants, ${ }^{5}$ selective serotonin reuptake inhibitor (SSRI) antidepressants ${ }^{6}$ and atypical antipsychotics. ${ }^{7}$ Despite a lack of guidelines for the use of antidepressant and antipsychotic medications in the treatment of eating disorders (EDs) like $\mathrm{AN}^{8}{ }^{9}$ they are sometimes prescribed to treat the symptoms associated with $\mathrm{AN}$ or to treat comorbidities, particularly if there is a less than optimal treatment response with first-line psychological treatments. ${ }^{10}$ A retrospective chart review study in the USA investigated the rates of psychopharmacological medication use in adolescents and young people in the USA who were referred to adolescent medicine-based ED programmes for the treatment of EDs. ${ }^{11}$ They found that at 1-year follow-up, psychopharmacological medications continue to be prescribed at a high rate $(58.7 \%$ compared with $20.4 \%$ at intake) in adolescents and young people with AN. A more recent study assessing 86 charts of patients with an ED in a tertiary care centre in the USA found $45.3 \%$ to be on psychotropic medications. ${ }^{12}$ Similarly, another study in the USA that aimed to investigate the use of psychotropic medications in women with AN between 1997 and 2009 found that the use of atypical antipsychotics had doubled during the study period from $8.9 \%$ to $18.5 \%$, while the use of antidepressants had remained stable. ${ }^{13}$

A small number of clinical trials and observational studies have aimed to focus on the safety and efficacy of atypical antipsychotics, specifically olanzapine, as adjuncts to the treatment of AN. ${ }^{14-18}$ One randomised controlled trial on eight patients receiving olanzapine at a dose range of $5-20 \mathrm{mg} /$ day and seven patients receiving chlorpromazine $25-200 \mathrm{mg}$ found that individuals on the olanzapine treatment had reduced anorexia thoughts, in comparison; however, no evidence was found to support BMI increases between the two groups. ${ }^{14}$ In contrast, a double-blind placebo controlled trial on 15 female patients with AN using olanzapine and cognitive behavioural therapy for 3 months and on 15 female patients with AN on placebo and CBT found no significant differences between the placebo group and olanzapine with regard to weight gain, but it was reported that in the binge-purge subtype of AN, significant differences in BMI were established. ${ }^{15}$ Similarly, in another doubleblinded placebo controlled trial in patients with AN who received a placebo and olanzapine at a mean dose of $6.61 \mathrm{mg} /$ day for 10 weeks, the olanzapine group showed a significant increase in weight gain rate, and $87.5 \%$ of the patients achieved complete weight restoration in comparison with $55.6 \%$ of the placebo group. ${ }^{16}$ The focus on olanzapine in clinical and research practice may be due to its favourable safety and efficacy profile derived from the daily practices of healthcare professionals, which encouraged researchers to conduct more studies with a focus on olanzapine to determine the evidence behind the prescribing practices of healthcare professionals. Based on this, it is important to explore the psychotropic drug prescribing practices of healthcare professionals, with emphasis on olanzapine prescribing by ED psychiatrists in a specialist secondary care setting for individuals with AN. This will provide a better understanding of AN pharmacotherapy in practice as there is currently no evidence base for prescribing antidepressants or antipsychotics for young people with EDs and can contribute to the current limited knowledge of current prescribing by providing a template for prescribing protocols for future research. Thus, this study aimed to explore contemporary prescribing practices of specialist CAED psychiatrists prescribing in a specialist secondary care setting for individuals with $\mathrm{AN}$, with special emphasis on olanzapine.

\section{METHODS}

An exploratory cross-sectional self-reported survey study was conducted using a questionnaire tool that was developed to explore three main areas: (1) the proportion of patients with AN prescribed psychotropic medications and the frequency of the medication reviews (pattern of psychopharmacological care); (2) the most common medications prescribed for young people with $\mathrm{AN}$, and the dosage and protocol for monitoring their side effects (medication treatment pattern); and (3) the process of continuing pharmacotherapy. The questionnaire had a total of 10 questions: 2 related to the pattern of psychopharmacological care provided by ED psychiatrists; 4 related to the medication treatment pattern; 3 related to pharmacotherapy continuation; and 1 related to consent for future studies (online supplementary material 1).

Content and face validity were checked by two senior consultant CAED psychiatrists (DN and MS), who assessed whether the questionnaire was likely to elicit the desired outcomes, and confirmed the content was readable and feasible and the layout and design were clear. The study was reported in accordance with Strengthening the Reporting of Observational Studies in Epidemiology statement (online supplementary material 2).

Questionnaires were distributed by two consultant CAED psychiatrists (DN and MS) to all CAED psychiatrists who attended the Health Education England national training held in the summer of 2017. These CAED psychiatrists represented CYP EDS in England. Questionnaires were collected at the end of the training day. Participants who did not return their questionnaires were followed up by email in order to obtain their response. The data were entered on a database independently by two reviewers ( $\mathrm{MB}$ and $\mathrm{KW}$ ), and results were compared to ensure homogeneity of data documentation. The data were then analysed and descriptive statistics were reported as frequencies and percentages using STATA V.14.

\section{Patient involvement}

Patients were not involved in the design of the study. 
RESULTS

A total of 44 CAED psychiatrists participated in the study from the 77 CYP EDS teams in England. As 13 of the CYP EDS had no psychiatrists in post at the time of the study, this accounted for $69 \%$ of child and adolescent psychiatrists treating children and young people with EDs in England who had attended the training day.

\section{Patterns of psychopharmacological care}

Psychiatrists were questioned in this survey to estimate the proportion of young people with AN under their care, for whom they prescribed psychotropic medications. Results are presented in table 1, with $40 \%$ of CAED psychiatrists estimating that under $10 \%$ of the individuals with AN in their service were prescribed psychotropic medications.

Nearly a third of CAED psychiatrists reported that they met with their patients for whom they have prescribed once a week during the first 18 weeks of treatment initiation. Majority of CAED psychiatrists $(38 \%)$ reported meeting their patients every 2 weeks, and $22 \%$ met their patients for a medication review once a month on average.

\section{Patterns of medication treatment for AN in children and adolescents}

Psychiatrists were asked about medications specifically prescribed for $\mathrm{AN}$, with olanzapine being the most commonly prescribed psychotropic medication for the treatment of children and adolescents with AN (38\% of psychiatrists). The second most commonly prescribed medication for AN was fluoxetine (29\%), followed by sertraline $(10 \%)$, non-specified SSRIs $(9 \%)$, risperidone and quetiapine $(4 \%)$, citalopram $(3 \%)$ and aripiprazole (1\%) (figure 1).

Prescribing olanzapine as first-choice psychotropic medication was reported by $40 \%$ of psychiatrists. This was followed by fluoxetine (35\%), non-specified SSRIs (15\%), sertraline $(8 \%)$ and risperidone (3\%). Similarly, as second choice for the prescription of psychotropic medication in young people with $\mathrm{AN}$, olanzapine was commonly selected by $33 \%$ of psychiatrists. Fluoxetine was selected by $22 \%$ and was closely followed by sertraline, with $19 \%$ of participants selecting it as second-choice medication. Other psychotropic medications prescribed as secondchoice treatment include quetiapine $(8 \%)$, risperidone $(6 \%)$, citalopram $(6 \%)$, aripiprazole $(3 \%)$ and non-specified SSRIs $(3 \%)$.

\section{Olanzapine treatment pattern}

The majority of psychiatrists (69\%) reported initiating olanzapine at a dose of $2.5 \mathrm{mg} /$ day. Initiation at a dose lower than $2.5 \mathrm{mg}$ /day was reported by $28 \%$ of psychiatrists, whereas a small percentage $(3 \%)$ reported initiating olanzapine at a dose higher than $2.5 \mathrm{mg}$ /day.

The starting dose of olanzapine was reported to be given for various durations before considering an increase or a reduction in the dose. The most common lengths of durations of olanzapine at the initial dose were less than a month (54\%) and 1-2 months (39\%). Only $7 \%$ of

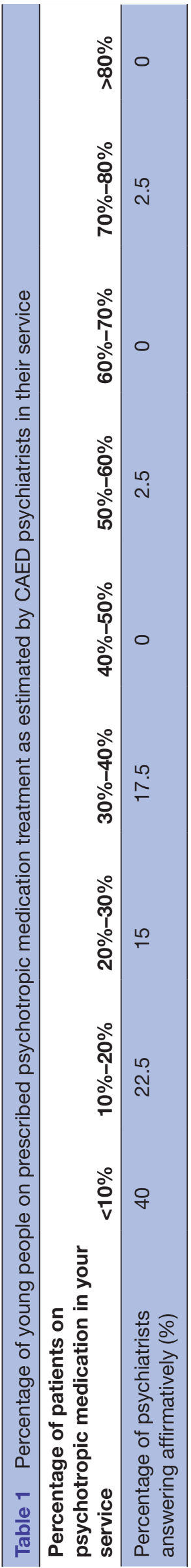




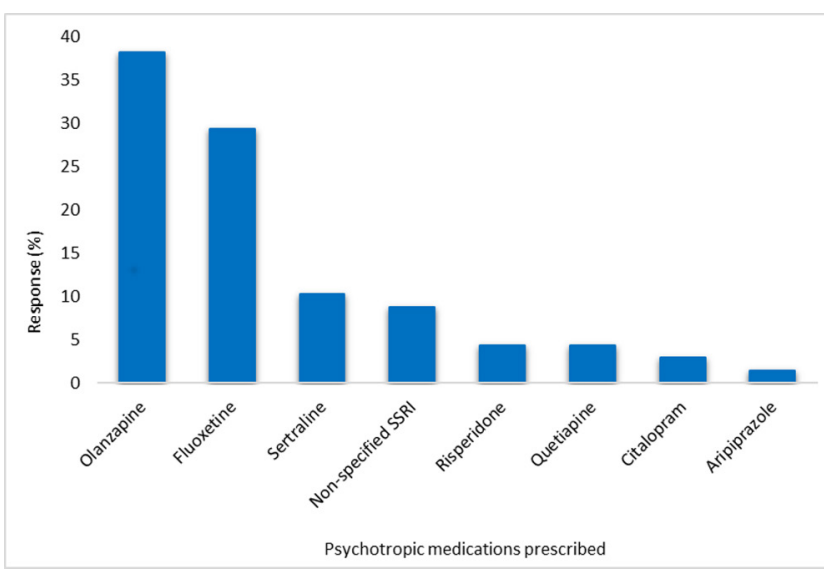

Figure 1 The preferred psychotropic medication prescribed for the treatment of anorexia nervosa. SSRI, selective serotonin reuptake inhibitor.

participants responded that they would give olanzapine at the initiation dose for more than 2 months.

Respondents were asked for information about their practice in escalating the dose and duration of olanzapine treatment in young people with AN. Over half of the psychiatrists $(58 \%)$ reported that they increase the dose in $2.5 \mathrm{mg}$ increments, while $35 \%$ stated that the incremental step in dosing was $1.25 \mathrm{mg}$ or less. Most psychiatrists reported increasing the dose of olanzapine in steps either every 2 weeks (35\%) or under 2 weeks $(30 \%)$, while $30 \%$ increased it every 2-4 weeks or monthly. Over half of the psychiatrists $(53 \%)$ responded that the maximum prescribed dose of olanzapine was $5 \mathrm{mg} /$ day. Similarly, $58 \%$ reported the total duration of olanzapine treatment to be under 6 months.

Majority of the psychiatrists $(76 \%)$ stated that a monitoring protocol or guidance is available for olanzapine use in young people with AN in their service, including laboratory tests (liver function, prolactin, HbAlc, lipids, and urea and electrolytes), anthropometrics (weight, height, body mass index and weight circumference), side effects experienced (sedation, drowsiness and metabolic side effects), symptoms experienced (eg, psychiatric) and cardiac outcome measurements (ECG, pulse and blood pressure).

\section{Patterns of olanzapine treatment continuation}

Almost all (98\%) of CAED psychiatrists reported that they inform general practitioners (GPs) about the treatment procedures undertaken with regards to olanzapine. Similarly, $97 \%$ of CAED psychiatrists reported that olanzapine prescribing is initiated from their service for the treatment of young people with $\mathrm{AN}$ rather than in primary care.

The findings for transferring the prescribing of olanzapine to the GPs to continue treatment varied between psychiatrists. Just over half of psychiatrists who responded to the survey $(51 \%)$ stated that they would not pass prescribing of olanzapine to the GPs. The reasons for not passing on olanzapine prescribing to GPs were as follows: the patients must be stabilised in order to pass prescribing, or the patient must be over the age of 16-18 years, or it will only be passed on if the treatment will be long term or the GP has declined to be involved with prescribing olanzapine to young people with AN. For the remainder, prescribing was on a shared care basis, with GPs prescribing with CAED psychiatrist oversight and clinical review.

\section{DISCUSSION}

This study has found that CAED psychiatrists self-report prescribing psychotropic medications for young people with AN. To the best of our knowledge, this is the first study to address pharmacotherapy approach by CAED psychiatrists, specifically olanzapine, for the treatment of young people with AN in England. There is currently a paucity of evidence in the use of psychotropic medications for AN, particularly in children and adolescents. This study highlights the need for further trial evidence for the use of medication in EDs and what doses of medications should be used.

The majority of CAED psychiatrists responded that under $10 \%$ of their patients were on psychotropic medications. This is significantly lower than what was reported in a US study that investigated the prescribing practice in EDs, which found that around $60 \%$ of patients with restrictive ED were prescribed psychotropic medication, most commonly SSRIs. ${ }^{11}$ In our English sample, only a few psychiatrists responded that the proportion of young people with AN on medication lies between $50 \%$ and $80 \%$. This difference could be due to the inclusion of restrictive ED in the US sample, which may consist of conditions other than just AN that are associated with higher rates of comorbidities. Cultural differences between prescribing practices in England and the USA must also be accounted for. In the US study, respondents were mainly adolescent medicine doctors (paediatricians) rather than psychiatrists, reflecting cultural differences in service organisations. In addition, practice guidelines are clearer on the lack of evidence for prescribing in England as stated by NICE. ${ }^{4}$

This study found that olanzapine is the most common psychotropic medication prescribed for the treatment of AN. This is in line with other studies conducted in the last decade. ${ }^{17} 18$ A previous UK study conducted on drug prescribing in CAED services using case notes found that 26 different drugs were used for AN treatment, with fluoxetine and olanzapine being the most widely used medications. ${ }^{10}$ However, large randomised controlled trials, which are needed to support evidence-based olanzapine prescribing, ${ }^{14151920}$ are lacking. The trials range in sample size from 15 to 152 patients in recent years and are not specific to the CAED population of interest.

In terms of prescribing practice, our findings were comparable with other studies for the most common initial olanzapine dosage and duration. CAED psychiatrists reported prescribing initial doses of $2.5 \mathrm{mg} /$ day 
for less than 1 month. Kafantaris et als study used olanzapine or a matching placebo, with an initial single oral dose of $2.5 \mathrm{mg} /$ day for 1 week. ${ }^{18}$ Similarly, Kafantaris $e t$ al reported increasing the dose of olanzapine by $2.5 \mathrm{mg}$ each week. Our study found that the majority of psychiatrists escalated treatment dosage and duration by $2.5 \mathrm{mg}$ increments every 2 weeks or less. This could be because the smallest available oral dose is $2.5 \mathrm{mg}$; thus, it is easier for psychiatrists to alter the dosage of treatment accordingly and more convenient for individuals with AN to comply with their psychotropic medications.

Our study found that the maximum reported dose of olanzapine prescription for young people with AN was $5 \mathrm{mg} /$ day, as reported by over half of the CAED psychiatrists $(53 \%)$. However, this result was contrary and lower to previous studies, which have reported a maximum target dose of $10 \mathrm{mg} /$ day during the duration of the studies. ${ }^{1618}$ Another study has reported the maximum olanzapine dose used as $7.5 \mathrm{mg}$ / day in 43 adolescent girls aged 10-17 years old, of whom 31 were diagnosed with AN. ${ }^{17}$ Similarly, Gowers et al found that the median dose of olanzapine prescribed was $7.5 \mathrm{mg}$ /day, with doses ranging from 1.2 to $20 \mathrm{mg}$ /day in some cases. ${ }^{10}$ These differences in dosage could be due to a lack in guidelines on psychotropic treatment in AN and are based on empirical judgements and experiences of the CAED psychiatrists.

One of the main strengths of this study is that it is the first questionnaire conducted among ED psychiatrists on pharmacotherapy in young people with AN. Thus, it provides a more detailed view on the prescribing practices of these individuals in England. Second, our study surveyed 69\% of CYP EDS in England, which reflects their practices on a national level. Third, our findings were based on real-life practices by surveying practising healthcare professionals.

Our study did not investigate why psychiatrists are prescribing psychotropic medications without any evidence to support their practices. This could be due to the lack of evidence for efficacy, as the literature primarily focused on diagnosis-based, rather than symptom-based prescribing of psychotropic medication. ${ }^{10}$ In practice, it is specific behaviours such as uncontrolled exercise and high emotional dysregulation that lead to the prescribing of psychotropic medications and not the diagnosis of AN alone. Furthermore, the reasoning behind prescribing psychotropics by CAED psychiatrists was not questioned, making the results harder to interpret as they may have been prescribed for psychiatric comorbidities in children and adolescents with AN and not solely for the ED itself. Further studies are needed to characterise the prescribing practices of psychiatrists and to explore the effects of these practices on patients' outcomes. In order to ensure that a good response rate was obtained for this study, this questionnaire was created in a short and simple format. As such, a limitation of this study was that we did not include open-ended questions, which would have allowed us to investigate barriers to and limitations and enhancing factors of pharmacotherapy in individuals with AN in more detail. Planned future works can include other qualitative study designs, such as interviews and focus groups, to enable us to generate thorough in-depth information. Similarly, as this was a self-reported survey, recall bias may be a contributing factor that must be acknowledged as it is not an entirely objective measure. In addition, social acceptability may have led to CAED psychiatrists overestimating aspects of the survey.

In conclusion, our study found that despite a lack of strong evidence, psychotropic medications, most commonly olanzapine, are prescribed for the treatment of young people with AN, either as an addition or alternative to psychotherapy. Future studies can be developed using a more detailed questionnaire study design, distributed among CAED psychiatrists, in order to attain further information on pharmacotherapy in young people.

Contributors MYB, ICKW, DN and MS contributed to the conception and design of the study. DN and MS carried out the collection of the data. The statistical analysis of the data was conducted by MYB and ICKW. MYB drafted the manuscript and was responsible for coordination of all aspects of the work. ICKW, DN, MS, RB and EM made substantial contributions to the drafts. Revision to the manuscript was made by MYB, DN and EM. All authors contributed to the interpretation of the results and the final manuscript.

Funding The authors have not declared a specific grant for this research from any funding agency in the public, commercial or not-for-profit sectors.

Competing interests None declared.

Patient consent for publication Not required.

Ethics approval Ethical approval was not required for this study as the research involved the use of a non-sensitive, completely anonymous educational survey; however, consent was obtained from all participating psychiatrists.

Provenance and peer review Not commissioned; externally peer reviewed.

Data availability statement Data are available upon reasonable request. All data relevant to the study are included in the article or uploaded as supplementary information.

Open access This is an open access article distributed in accordance with the Creative Commons Attribution Non Commercial (CC BY-NC 4.0) license, which permits others to distribute, remix, adapt, build upon this work non-commercially, and license their derivative works on different terms, provided the original work is properly cited, appropriate credit is given, any changes made indicated, and the use is non-commercial. See: http://creativecommons.org/licenses/by-nc/4.0/.

\section{REFERENCES}

1. Pisetsky EM, Utzinger LM, Peterson CB. Incorporating social support in the treatment of anorexia nervosa: special considerations for older adolescents and young adults. Cogn Behav Pract 2016;23:316-28.

2. Balestrieri M, Oriani MG, Simoncini A, et al. Psychotropic drug treatment in anorexia nervosa. search for differences in Efficacy/ Tolerability between adolescent and Mixed-age population. European Eating Disorders Review 2013;21:361-73.

3. Shih P-anB. Integrating multi-omics biomarkers and postprandial metabolism to develop personalized treatment for anorexia nervosa. Prostaglandins Other Lipid Mediat 2017;132:69-76.

4. NICE NIfHaCE. Eating disorders: recognition and treatment, 2018. Available: https://www.nice.org.uk/guidance/ng69 [Accessed 12 Oct 2018].

5. KKC M, Ip P, Hsia Y, et al. Adhd drug prescribing trend is increasing among children and adolescents in Hong Kong. Journal of attention disorders 2017;21:1161-8.

6. Murray ML, de Vries CS, Wong IC. A drug utilisation study of antidepressants in children and adolescents using the general practice research database. Arch Dis Child 2004;89:1098-102. 
7. Rani F, Murray ML, Byrne PJ, et al. Epidemiologic features of antipsychotic prescribing to children and adolescents in primary care in the United Kingdom. Pediatrics 2008;121:1002-9.

8. Couturier J, Lock J. A review of medication use for children and adolescents with eating disorders. J Can Acad Child Adolesc Psychiatry 2007;16:173-6.

9. McKnight RF, Park RJ. Atypical antipsychotics and anorexia nervosa: a review. European Eating Disorders Review 2010;18:10-21.

10. Gowers S, Claxton M, Rowlands L, et al. Drug prescribing in child and adolescent eating disorder services. Child Adolesc Ment Health 2010;15:18-22.

11. Monge MC, Forman SF, McKenzie NM, et al. Use of Psychopharmacologic medications in adolescents with restrictive eating disorders: analysis of data from the National eating disorder quality improvement collaborative. Journal of Adolescent Health 2015;57:66-72.

12. Mizusaki K, Gih D, LaRosa C, et al. Psychotropic usage by patients presenting to an academic eating disorders program. Eating and Weight Disorders - Studies on Anorexia, Bulimia and Obesity 2018;23:769-74.

13. Fazeli PK, Calder GL, Miller KK, et al. Psychotropic medication use in anorexia nervosa between 1997 and 2009. International Journal of Eating Disorders 2012;45:970-6.
14. Mondraty N, Laird Birmingham C, Touyz S, et al. Randomized controlled trial of olanzapine in the treatment of cognitions in anorexia nervosa. Australasian Psychiatry 2005;13:72-5.

15. Brambilla F, Garcia CS, Fassino S, et al. Olanzapine therapy in anorexia nervosa: psychobiological effects. Int Clin Psychopharmacol 2007;22:197-204.

16. Bissada H, Tasca GA, Barber AM, et al. Olanzapine in the treatment of low body weight and obsessive thinking in women with anorexia nervosa: a randomized, double-blind, placebo-controlled trial. American Journal of Psychiatry 2008;165:1281-8.

17. Norris ML, Spettigue W, Buchholz A, et al. Olanzapine use for the adjunctive treatment of adolescents with anorexia nervosa. $J$ Child Adolesc Psychopharmacol 2011;21:213-20.

18. Kafantaris V, Leigh E, Hertz S, et al. A placebo-controlled pilot study of adjunctive olanzapine for adolescents with anorexia nervosa. $J$ Child Adolesc Psychopharmacol 2011;21:207-12.

19. Mehler-Wex C, Romanos M, Kirchheiner J, et al. Atypical antipsychotics in severe anorexia nervosa in children and adolescents - review and case reports. European Eating Disorders Review 2008;16:100-8.

20. Attia E, Steinglass JE, Walsh BT, et al. Olanzapine versus placebo in adult outpatients with anorexia nervosa: a randomized clinical trial. American Journal of Psychiatry 2019;176:449-56. 